\title{
Gamma-ray emission from early-type stars interacting with AGN jets
}

\author{
Anabella T. Araudo1,a, Valentí Bosch-Ramon², and Gustavo E. Romero ${ }^{3,4}$ \\ ${ }^{1}$ Centro de Radioastronomía y Astrofísica, Universidad Nacional Autónoma de México, A.P. 3-72 (Xangari), 58089 Morelia, \\ Michoacán, México. \\ ${ }^{2}$ Universitat de Barcelona. Departament d'Astronomia i Meteorologia Marti i Franques 1, 7th floor ES 08028 Barcelona, Spain. \\ ${ }^{3}$ Instituto Argentino de Radioastronomía, C.C.5, (1894) Villa Elisa, Buenos Aires, Argentina. \\ ${ }^{4}$ Facultad de Ciencias Astronómicas y Geofísicas, Universidad Nacional de La Plata, Paseo del Bosque, 1900 La Plata, Argentina.
}

\begin{abstract}
We study the interaction of early-type stars with the jets of active galactic nuclei. A bow-shock will form as a consequence of the interaction of the jet with the winds of stars and particles can be accelerated up to relativistic energies in these shocks. We compute the non-thermal radiation produced by relativistic electrons from radio to gamma-rays. This radiation may be significant, and its detection might yield information on the properties of the stellar population in the galaxy nucleus, as well as on the relativistic jet. This emission is expected to be relevant for nearby non-blazar sources.
\end{abstract}

\section{Introduction}

Active galactic nuclei (AGNs) consist of a supermassive black hole $(\mathrm{SMBH})$ surrounded by an accretion disc in the center of a galaxy. Sometimes these objects present radio emitting jets originated close to the SMBH and ejected perpendicular to the accretion disc. Radio-loud AGNs present thermal and non-thermal continuum emission in the whole electromagnetic spectrum, from radio to gamma-rays.

In the nuclear region of AGNs there is matter in the form of diffuse gas, clouds, and stars, making jet medium interactions likely [1]. We study the interaction of massive stars with the AGN jets $[4,7]$. We adopt the main idea of [5], i.e. the interaction of massive stars with AGN jets, although our scenario consists of a population of massive stars surrounding the jets, and considers jet-star interactions at different heights $(z)$ of the jet. We analyze the dependence with $z$ of the properties of the interaction region (i.e. the shocks in the jet and the stellar wind), and also the subsequent non-thermal processes generated at these shocks. We consider the injection of relativistic electrons, the evolution of this population of particles by synchrotron and inverse Compton (IC) radiation, as well as escape losses.

In the scenario considered here, the emitters are the flow downstream of the bow shocks located around the stars. This flow moves together with the stars at a nonrelativistic speed, and thus the emission will not be relativistically boosted. For this reason the radiation from jet-star interactions will be mostly important in misaligned AGNs, where the emission produced by other mechanisms in the jet is not amplified by Doppler boosting. In the GeV domain, the Fermi satellite has already detected at least 11 misaligned radio-loud AGNs, a population that is expected to grow in the near future. Because of this, theoretical models that can predict the level and spectrum of the gamma-ray emission from these sources are desirable to contribute to the understanding of future detections.

\section{Stellar populations in AGNs}

The number of stars formed per unit of mass $(m)$, time $(t)$ and volume $\left(V \propto r^{3}\right)$ is $\psi(m, r, t)=\psi_{0}(m, r) \exp (-t / T)$, where $t$ and $T$ are the age of the stellar system and the duration of the formation process, respectively. We consider that stellar formation processes take place continuously $(t \ll T)$ in the nuclear region of the galaxy, and the stars are uniformelly distributed around the SMBH. We assume that

$$
\psi=K\left(\frac{m}{M_{\odot}}\right)^{-2.3}\left(\frac{r}{\mathrm{pc}}\right)^{-2}
$$

where $0.1 \leq m / M_{\odot} \leq 120$. (In [3], $\psi \propto r^{-1}$ is also considered.) The star formation rate is $\dot{M}_{\star}=\iint \psi m \mathrm{~d} m \mathrm{~d} V$, i.e.:

$$
\dot{M}_{\star}=K \int_{1 \mathrm{pc}}^{1 \mathrm{kpc}}\left(\frac{r}{\mathrm{pc}}\right)^{-2} 4 \pi r^{2} \mathrm{~d} r \int_{0.1 M_{\odot}}^{120 M_{\odot}}\left(\frac{m}{M_{\odot}}\right)^{-1.3} \mathrm{~d} m .
$$

We integrate Eq. 2 from 1 pc to $1 \mathrm{kpc}$, which is the maximun value where the following empirical relation is valid [9]:

$$
\frac{\dot{M}_{\star}}{\mathrm{M}_{\odot} \mathrm{yr}^{-1}} \sim 47.86\left(\frac{\dot{M}_{\mathrm{bh}}}{\mathrm{M}_{\odot} \mathrm{yr}^{-1}}\right)^{0.89} \sim 714 \eta_{\mathrm{acc}}^{0.89} .
$$

\footnotetext{
ae-mail: a.araudo@crya.unam.mx
} 


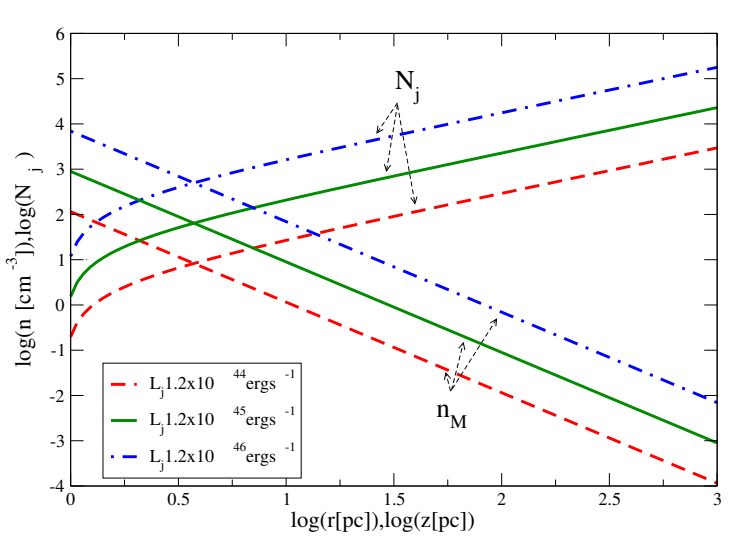

Figure 1. Density of massive stars $\left(n_{\star \mathrm{M}}\right)$ and number of massive stars inside the jet $\left(N_{\star \mathrm{j}}\right)$ for the different values of $z$. Cases for different values of $\eta_{\text {acc }}$ are plotted.

In the previous equation we have used that the SMBH accretion rate, $\dot{M}_{\mathrm{bh}}$, is related with the SMBH mass, $M_{\mathrm{bh}}$, as $0.1 \dot{M}_{\mathrm{bh}} c^{2}=\eta_{\mathrm{acc}} L_{\mathrm{Edd}}$, where $L_{\mathrm{Edd}}=1.2 \times$ $10^{47}\left(M_{\mathrm{bh}} / 10^{9} M_{\odot}\right) \mathrm{erg} \mathrm{s}^{-1}$ is the Eddington luminosity. We fix $M_{\mathrm{bh}}=10^{9} \mathrm{M}_{\odot}$, and $\eta_{\mathrm{acc}}=0.01,0.1$, and 1. (In [3] different values of $M_{\mathrm{bh}}$ are considered.) Then, from Eqs. (2) and (3), $K$ results $\sim 0.01 \eta_{\text {acc }}^{0.89}$.

Once a stellar population is injected in the host galaxy, stars of a given mass are accumulated in the galaxy and, at a time $t<t_{\text {life }}$, where $t_{\text {life }}=a\left(m / M_{\odot}\right)^{-b}$ Gyr is the stellar lifetime, the density of stars (per unit of mass) is $n_{\star m} \approx \psi t$. For $t>t_{\text {life }}$, stars die and the mass distribution follows a law $n_{\star \mathrm{m}} \propto m^{-(2.3+b)}$. In the case of massive stars, $(a, b)=(1,1.7)$ and $(0.1,0.7)$ for $m<15 \mathrm{M}_{\odot}$ and $60>m / M_{\odot}>15$, respectively [6]. For $m>60 \mathrm{M}_{\odot}$, $t_{\text {life }} \sim 0.004$ Gyr. Then, at $t \gtrsim t_{\text {life }}\left(8 M_{\odot}\right) \sim 0.03 \mathrm{Gyr}$, the rate of stellar formation is equal to the rate of stellar death and the system reaches the steady state for $m>8 M_{\odot}$. In such a case, the number density of massive stars $-n_{\star \mathrm{M}^{-}}$ keeps the spatial dependence of the stellar injection rate, $\psi \propto r^{-2}$, resulting

$$
\frac{n_{\star \mathrm{M}}}{\mathrm{pc}^{3}}=\int_{8 M_{\odot}}^{120 M_{\odot}} n_{\star \mathrm{m}} \mathrm{d} m \sim 6.9 \times 10^{3} \eta_{\mathrm{acc}}^{0.89}\left(\frac{r}{\mathrm{pc}}\right)^{-2}
$$

as is shown in Fig. 1.

The number of massive stars contained in the jet volume is $N_{\star \mathrm{j}}(z)=\int_{1 \mathrm{pc}}^{z} n_{\star \mathrm{M}}\left(z^{\prime}\right) \mathrm{d} V_{\mathrm{j}}$, where $\mathrm{d} V_{\mathrm{j}}=\pi R_{\mathrm{j}}^{2} \mathrm{~d} z^{\prime} \quad(z$ is the $r$-coordinate along the jet and the jet radius $R_{\mathrm{j}}$ is $\sim 0.1 z)$. This yields:

$$
N_{\star \mathrm{j}} \sim 8.6 \times 10^{4} \eta_{\mathrm{acc}}^{0.89}\left[\left(\frac{z}{\mathrm{pc}}\right)-1\right] .
$$

Note that at $z \geq z_{1} \sim \eta_{\mathrm{acc}}^{-0.89} \mathrm{pc}$ there is at least one massive star inside the jet at every time (see Fig. 1).

\section{Jet-star interaction}

We consider massive stars with mass loss rate and terminal wind velocity $\dot{M}_{\mathrm{w}}=10^{-6} \mathrm{M}_{\odot} \mathrm{yr}^{-1}$ and $v_{\infty}=2000 \mathrm{~km} \mathrm{~s}^{-1}$, respectively. When the jet interacts with stars a double bow shock is formed around them. The location of the stagnation point is at a distance $R_{\mathrm{sp}}$ from the stellar surface, where the wind and jet ram pressures are equal. From $\rho_{\mathrm{w}} v_{\infty}^{2}=\rho_{\mathrm{j}} c^{2} \Gamma$, where $\rho_{\mathrm{w}} \sim \dot{M}_{\star} /\left(4 \pi R_{\mathrm{sp}}^{2} v_{\infty}\right)$ is the wind density, we obtain

$\frac{R_{\mathrm{sp}}}{R_{\mathrm{j}}} \sim 10^{-3}\left(\frac{\dot{M}_{\mathrm{w}}}{10^{-6} \mathrm{M}_{\odot} / \mathrm{yr}}\right)^{1 / 2}\left(\frac{v_{\infty}}{2000 \mathrm{~km} / \mathrm{s}}\right)^{1 / 2}\left(\frac{L_{\mathrm{j}}}{10^{44} \mathrm{erg} / \mathrm{s}}\right)^{-1 / 2}$,

resulting $R_{\mathrm{sp}} \propto z$. We assume that the jet has a Lorentz factor $\Gamma=10$ and a velocity $\sim c$. The jet kinetic luminosity is determined as $L_{\mathrm{j}}=\eta_{\mathrm{j}} L_{\mathrm{Edd}}$, giving $L_{\mathrm{j}}=1.2 \times 10^{44}, 1.2 \times$ $10^{45}$, and $1.2 \times 10^{46} \mathrm{erg} \mathrm{s}^{-1}$, for $\eta_{\mathrm{j}}=0.001,0.01$, and 0.1 , respectively. The jet density is obtained as $\rho_{\mathrm{j}}=L_{\mathrm{j}} /[(\Gamma-$ 1) $\left.c^{3} \pi R_{\mathrm{j}}^{2}\right]$.

\section{Particle acceleration}

Electrons are accelerated up to relativistic energies in both the jet and wind shocks, and injected in the downstream regions following a distribution $Q_{e} \propto E_{e}^{-2.2}$. Under the assumption of a one-zone model for the accelerator/emitter, we solve

$$
\frac{N_{e}}{t_{\mathrm{esc}}}-\frac{\mathrm{d}}{\mathrm{d} E_{e}}\left(\dot{E}_{e} N_{e}\right)=Q_{e}
$$

to derive the energy distribution of relativistic electrons $N_{e}$, where $t_{\mathrm{esc}}=\min \left\{t_{\mathrm{adv}}, t_{\mathrm{diff}}\right\}$. The diffusion timescale is $t_{\mathrm{diff}} \sim D_{\mathrm{j}, \mathrm{w}}^{2} q B_{\mathrm{jbs}, \mathrm{wbs}} /\left(E_{e} c\right)$ in the Bohm regime, where $B_{\mathrm{jbs}}$ and $B_{\text {wbs }}$ are the magnetic fields in the jet and the stellar wind bow-shock regions, respectively, and $q$ is the electron charge. The advection escape times in the downstream regions of the jet and the wind bow shocks are $t_{\mathrm{adv}, \mathrm{j}} \sim 3 R_{\mathrm{sp}} / c$ and $t_{\mathrm{adv}, \mathrm{w}} \sim 4 R_{\mathrm{sp}} / v_{\infty}$, respectively. Besides escape losses, electrons suffer synchrotron and stellar photon IC upscattering losses, $\dot{E}_{e}$. For the later we have considered stellar target photons with an energy and luminosity $\sim 30 \mathrm{eV}$ and $L_{\star}=3 \times 10^{38} \mathrm{erg} \mathrm{s}^{-1}$, respectively. For synchrotron and diffussion we have to estimate $B_{\mathrm{jbs}}$ and $B_{\mathrm{wbs}}$.

Assuming that the magnetic energy density in the jet is a fraction 0.3 of its kinetic energy density [8], and that in the shocked region the magnetic field is amplified by a factor of 4, we obtain

$$
B_{\mathrm{jbs}} \sim\left(\frac{L_{\mathrm{j}}}{10^{44} \mathrm{erg} \mathrm{s}^{-1}}\right)^{1 / 2}\left(\frac{z}{\mathrm{pc}}\right)^{-1} \mathrm{G} .
$$

With this value of $B_{\mathrm{jbs}}$, the maximum energy achieved by electrons in the jet bow shock is determined by synchrotron losses, giving $E_{e}^{\max } \sim$ $1.2 \times 10^{2}(z / \mathrm{pc})^{1 / 2}\left(L_{\mathrm{j}} / 10^{44} \mathrm{erg} \mathrm{s}^{-1}\right)^{-1 / 4} \mathrm{TeV}$. For the wind we assume the parametrization of the magnetic field given in [10], with a value in the stellar surface of about $10 \mathrm{G}$. The maximum energies for electrons accelerated in the wind bow shock are determined by IC scattering and diffussion (see Fig. 2). 


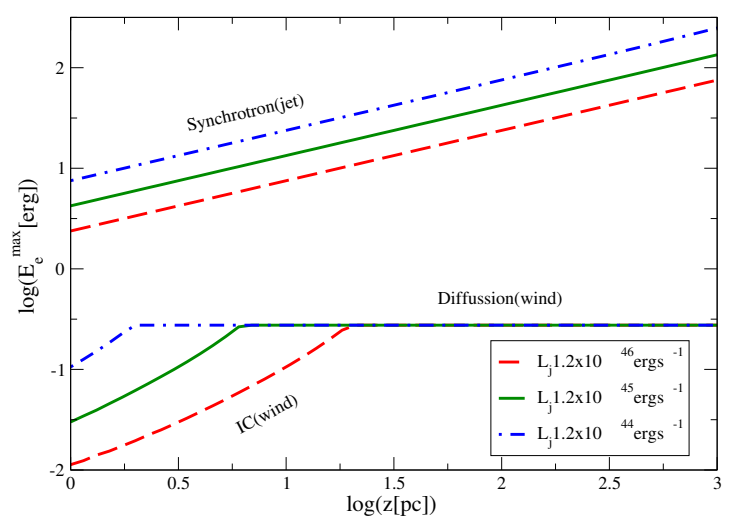

Figure 2. Maximum energies of electrons accelerated in the jet (top) and wind (bottom) bow shocks at different $z$.

Taking into account the escape, synchrotron, and IC losses described above, we solve Eq. (7) obtaining the energy distribution $N_{e}$ of relativistic electrons in the jet and in the wind. In the former, synchrotron and IC cooling dominates a significant part of the electron energy distribution up to a certain height, at which advection losses become dominant. (However, $E_{e}^{\max }$ in the jet is always constrained by synchrotron cooling.) This is due to the different $z$-dependence of these timescales, $z^{2}$ for synchrotron and IC, and $z$ for advection. In the later, synchrotron and IC cooling dominates a significant part of the spectrum, but at large values of $z$ diffusion losses become dominant all the way up to $E_{e}^{\max }$. In both cases, Thomson IC and synchrotron dominance appear as a steepening in $N_{e}$ from $\propto E_{e}^{-2.2}$ to $\propto E_{e}^{-3.2}$.

\section{Non-thermal emission}

Once $N_{e}$ in the jet and wind shocked regions is computed, we calculate the spetral energy distribution (SED) of the non-thermal radiation, synchrotron and IC scattering (in Thomson and Klein-Nishina regimes) in the jet and the wind shocked regions, using the standard fomulae. The energy budget for the emission produced in the bow shock regions are $\sim \eta_{\mathrm{nt}}\left(R_{\mathrm{sp}} / R_{\mathrm{j}}\right)^{2} L_{\mathrm{j}}$ and $\eta_{\mathrm{nt}} L_{\mathrm{w}} / 4$, where $L_{\mathrm{w}}=\dot{M}_{\mathrm{w}} v_{\infty}^{2} / 2$ and $\eta_{\mathrm{nt}}$ is fixed in 0.1. An important characteristic of the jet/star scenario is that the emitter is fixed to the star, and being the star moving at a non-relativistic velocity, the emission produced in the bow shock regions is not amplified by Doppler boosting.

As is shown in Fig. 3, the emission produced by massive stars per interaction at small values of $z$ is higher than emission produced at larger $z$, as a consequence of the dilution of the target fields (the photon density decreases as $z^{-2}$ and $B_{\mathrm{jbs}}$ as $z^{-1}$ ). Synchrotron emission produced in the jet bow-shock is more than 100 times larger than the emission produced in the wind, but IC radiation generated in the jet and in the wind both reach the similar luminosity along $z$.
However, at large values of $z$ the number of stars interacting with the jet is $>1$ and the emission produced by all of them increases with $z$. We calculate the emission produced by each one of the massive stars located inside the jet at each $z$ and then we integrate along $z$ all the contributions, as is shown in Fig. 4. The bolometric luminosities plotted in the figure correspond to the total emission produced by each jet/star interactions, i.e. it is the sum of the bolometric luminosity produced both in the jet and in the wind, by synchrotron and IC emission. Note that the bolometric luminosities produced by only one star interacting with the jet at each $z$ are small. However, when we consider that $N_{\star \mathrm{j}}$ stars are inside the jet, the bolometric luminosities produced by all of them is significantly larger.

\subsection{Gamma-ray emission}

From Fig. 3 we can see that most of the energy radiated by jet/star interactions at any $z$ is in the gamma domain, in particular in the case of the wind emission. The jet emission at $z \gtrsim 10 \mathrm{pc}$ is dominated by synchrotron radiation, but at $z \lesssim 10$ pc synchrotron and IC emission levels are similar. In the cases with low values of $N_{\mathrm{j} \star}\left(\eta_{\mathrm{acc}}=0.01\right)$, the produced high-energy emission can not be detected by Fermi satellite (in the range $0.1-1 \mathrm{GeV}$ ). The most interesting cases are those with $\eta_{\text {acc }}=0.1$ and 1 , whose emission could be marginally detectable in the case of sources located at a distance $\lesssim 50 \mathrm{Mpc}$. We note that the emission produced by jet/star interactions will be more prominent in AGNs with dense stellar populations. In particular, the interaction of a star forming region with a jet will be study by us in a future work.

Given the typical stellar photon energy $E_{0} \sim 10 \mathrm{eV}$, gamma rays beyond $\sim 30 \mathrm{GeV}$ can be affected by photonphoton absorption due to the presence of the stellar radiation field. However, this process is only important at $z<1 \mathrm{pc}$, where $R_{\mathrm{sp}}$ is small. Another effect that should be considered at energies beyond $100 \mathrm{GeV}$ is absorption in the extragalactic background light via pair creation (important only for sources located well beyond $100 \mathrm{Mpc}$ ).

\section{Discussion}

We have studied the interaction of massive stars with AGN jets, focusing on the production of gamma rays from particles accelerated in the double bow-shock structure formed around the stars as a consequence of the jet/stellar wind interaction. We calculated the energy distribution of electrons accelerated in the jet and in the wind, and the subsequent non-thermal emission from these relativistic particles. In the jet and wind shocked regions, the most relevant radiative processes are synchrotron emission and IC scattering of stellar photons. The properties of the emission generated in the downstream region of the bow shocks change with $z$. On the one hand, the target densities for radiative interactions decrease as $z^{-2}$. On the other hand, the time of the non-thermal particles inside the emitter is $\propto R_{\mathrm{sp}} \propto z$, and the number of stars per jet length unit $\mathrm{d} N_{\star, \mathrm{j}} / \mathrm{d} z \propto z$. Therefore, for a population of stars, the last 

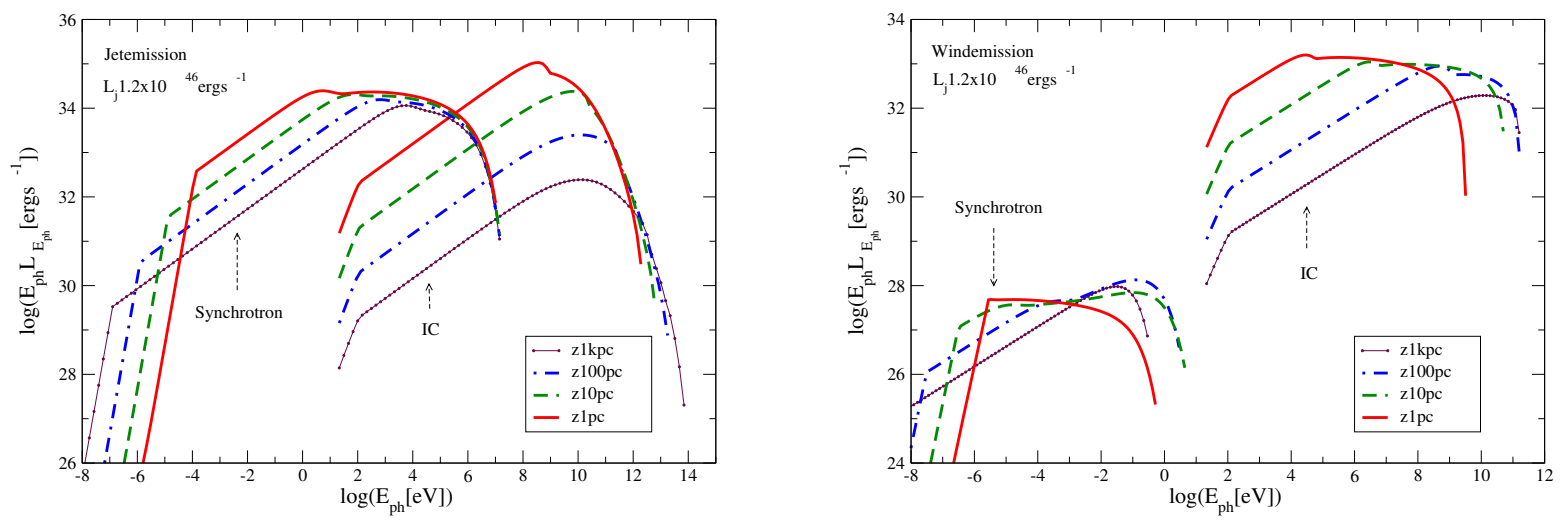

Figure 3. Spectral energy distribution produced in the jet (left) and in the wind (right) by the interaction of only one star with a jet of $L_{\mathrm{j}}=1.2 \times 10^{46} \mathrm{erg} \mathrm{s}^{-1}$ at different $z$.

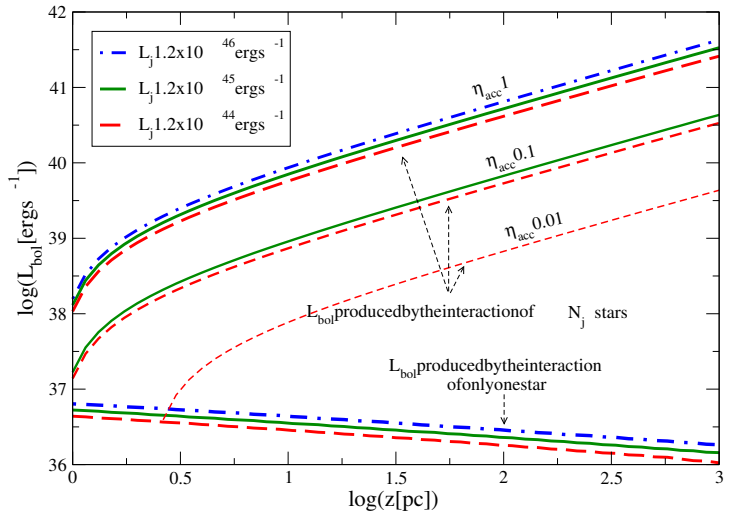

Figure 4. Total bolometric luminosities at different $z$.

two effects soften the emission drop with $z$. In the case of $M_{\mathrm{bh}}=10^{9} \mathrm{M}_{\odot}$, and high accretion rates $\left(\eta_{\mathrm{acc}}=1\right)$, gamma-ray luminosities $\sim 10^{41} \mathrm{erg} \mathrm{s}^{-1}$ may be achieved (see Fig. 4). However, note that few powerful Wolf-Rayet stars inside the jet could actually dominate over the whole main-sequence OB star population $[2,3]$.

Since jet-star emission should be rather isotropic, it would be masked by jet beamed emission in blazar sources. Although radio loud AGN jets do not display significant beaming, these objects may emit gamma-rays from jet/star interactions. Misaligned AGNs represent an increasing population of $\mathrm{GeV}$ sources, as is shown is the second catalog of the Fermi satellite. Close and powerful sources could be detectable by deep enough observations of Fermi. After few-year exposure times, a significant signal from these objects could be found, and their detection can shed light on the jet matter composition as well as on the stellar populations in the vicinity of AGNs.

\section{Acknowledgments}

This project is financially supported by PAPIIT, UNAM; PIP 0078/2010 from CONICET, and PICT 848/2007 (Argentina). G.E.R. and V.B-R. acknowledge support from grants AYA 2010-21782-C03-01 and FPA2010-22056C06-02.

\section{References}

[1] Araudo, A. T.; Bosch-Ramon, V.; Romero, G. E.; A\&A 522, 97 (2010)

[2] Araudo, A. T.; Bosch-Ramon, V.; Romero, G. E.; AIPC 1505, 614-617 (2012)

[3] Araudo, A. T.; Bosch-Ramon, V.; Romero, G. E.; MNRAS (submitted)

[4] Barkov, M. V.; Aharonian, F. A.; Bosch-Ramon, V.; ApJ 724, 1517-1523 (2010)

[5] Bednarek, W.;Protheroe, R. J.; MNRAS 287, L9-L13 (1997)

[6] Ekström, S.; Georgy, C.; Eggenberger, P. et al.; A\&A 537, 146-164 (2012)

[7] Komissarov, S. S., MNRAS 269, 394 (2004)

[8] Komissarov, S. S; Barkov, M. V.; Vlahakis, N.; Königl, A; MNRAS 380, 51-70 (2007)

[9] Satyapal, S.; Dudik, R. P.; O'Halloran, B. et al; ApJ 633, 86-104 (2005)

[10] Usov, V. V.; Melrose, D. B.; ApJ 395, 575-581 (1992) 